\title{
Correlative Confocal and Environmental Scanning Electron Microscopy for Investigating the Fungal Invasion of Plant Surfaces in Their Native State
}

\author{
Andrew J. Bowling ${ }^{1}$, Heather E. Pence ${ }^{1}$, Thomas Slanec ${ }^{1}$, and Leah L. Granke ${ }^{1}$ \\ ${ }^{1 .}$ Dow AgroSciences, Indianapolis, IN, 46268
}

Zymoseptoria tritici (wheat leaf blotch) can cause up to 50\% yield losses in untreated wheat in Europe. Fungicide applications can significantly reduce yield losses, but fungicide resistance has been documented [1]. Hence, new fungicides are necessary for long-term management of this disease. Most fungicidally active molecules inhibit fungal spore germination and/or germ tube growth. The ability to visualize the impact of active molecules on fungal structures growing on wheat leaves would greatly facilitate the development of new fungicides. However, imaging fungal structures in situ is non-trivial due to the fixation, dehydration, and critical point drying required for conventional high-vacuum SEM. Development of an imaging method that requires less sample preparation but still allows visualization of fungal infections would improve our understanding of this disease and aid development of future treatments and disease management strategies.

Environmental SEM (ESEM) allows the imaging of fully hydrated biological samples, thus facilitating the observation of fungal structures on plant surfaces in their native state. In order to acquire high-quality ESEM images of wheat leaves infected by Z. tritici, several factors were tested and optimized. We found that fungal spores freshly harvested from diseased leaves were much more regular in size and shape than those grown on potato dextrose agar. The density of spores applied to leaves was also a key variable, as the density should be high enough to be able to find spores easily in the microscope, but low enough that the spores don't aggregate together and obscure details. Wheat seedling leaves have a naturally ultrahydrophobic surface [2], so the inclusion of a surfactant was critical to allow the inoculum droplet to stick to, and spread on, the leaf surface. Several common surfactants were tested for compatibility with ESEM imaging, and although all of the surfactants tested appeared to work to some extent, we found that Tween $^{\text {TM }} 20$ yielded the highest quality images (Figure 1).

A lower-magnification light microscopic view of infected leaf surfaces gives a broader overview of the biology of the host/pathogen interaction. In order to find the relatively small $(25 \times 1.5 \mu \mathrm{m})$ spores on the leaf surface by light microscopy, it was necessary to use a GFP-expressing culture and fluorescence microscopy. Unfortunately, the large height variation between the ridges and valleys of a wheat leaf made it impossible to keep fine fungal structures in focus using widefield fluorescence microscopy. Because the optics of the confocal microscope physically exclude out-of-focus light from the sample, this instrument is ideal for collecting depth series images. A method was developed for using confocal microscopy directly of leaf surfaces without any mountant or coverslip (Figure 2A).

Because this confocal microscopy imaging method is non-destructive, it was possible to image a leaf first by low-magnification confocal microscopy, then image the same region at higher magnification by ESEM, and digitally correlate the images (Figure 2). The multi-color capability of the confocal microscope could be used to differentiate features, which would then be imaged at high magnification by ESEM. This correlative method is well-suited for studies where a specific feature of the leaf/fungus interaction is rare or hard to find. This technique allows for correlation of low-magnification confocal images with fine EMlevel details to view fungus/leaf interactions at multiple scales. 
[1] H Fones and S Gurr, Fungal Gen. and Biol. 79 (2015), p. 3.

[2] JH Troughton and DM Hall, Aust. J. Biol. Sci. 20 (1967), p. 509.
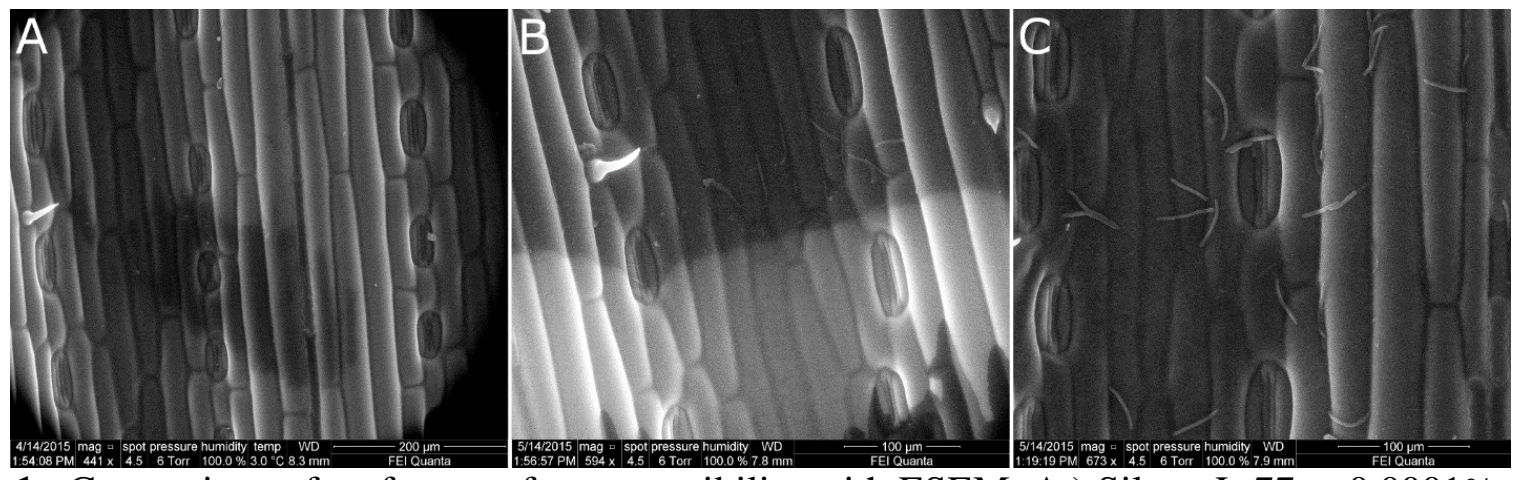

Figure 1. Comparison of surfactants for compatibility with ESEM. A.) Silwet L-77 at $0.0001 \%$ v/v. B.) Triton-X 100 at $0.005 \%$ v/v. C.) Tween 20 at $0.005 \%$ v/v.
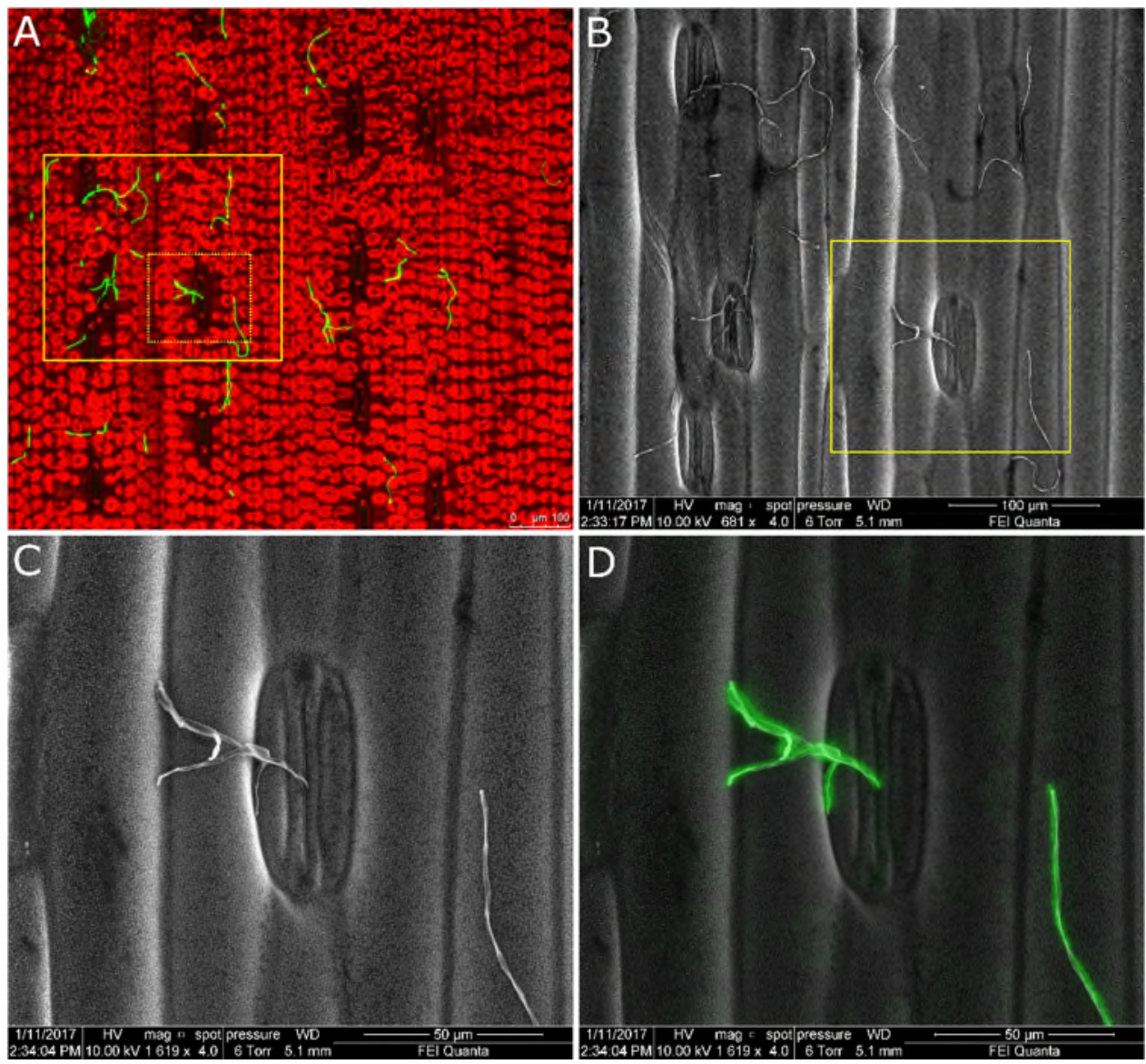

Figure 2. Confocal and ESEM images of the same region of a wheat leaf infected with Z. tritici. A.) A maximum-projection image of a confocal image stack of a wheat leaf surface infected with GFPexpressing Z. tritici. B.) An ESEM image of the region within the solid yellow box in (A). C.) A higher magnification ESEM image of the boxed region in (B) and the dashed box in (A). D.) An overlay of the fluorescence image in (A) over the high magnification image shown in (C). 